\title{
Pasión por el Trabajo: Evidencias de Validez Discriminante, Predictiva e Incremental
}

\author{
Solana Salessi \\ Consejo Nacional de Investigaciones Científicas \\ y Técnicas, Argentina.
}

\author{
Alicia Omar \\ Consejo Nacional de Investigaciones \\ Científicas y Técnicas, Argentina.
}

Resumen: El objetivo de este estudio fue establecer la validez discriminante, predictiva e incremental de la pasión por el trabajo respecto a los constructos de flow, implicación y satisfacción laboral, respectivamente. Se realizó un estudio empírico-instrumental con una muestra no probabilística (510 trabajadores, 54\% mujeres). Análisis de ecuaciones estructurales exploratorios (ESEM) mostraron saturaciones cruzadas inferiores a $.30 \mathrm{y}$ un adecuado ajuste de un modelo hepta-factorial oblicuo (GFI $=0,92, \mathrm{CFI}=0,90, \mathrm{TLI}=.91$, $\mathrm{RMSEA}=0,04$ ). Los valores de varianza media extraída, de su raíz cuadrada y de la proporción heterorasgo-monorasgo indicaron capacidad de discriminación entre los constructos (AVE $>0,50 ; \sqrt{ } \mathrm{AVE}<r$, HTMT $<0,90$ ). Análisis de regresión múltiple jerárquica confirmaron la contribución significativa de la pasión en la explicación de la satisfacción (pasión armoniosa: $\beta=.49, t=10.96, p=0,000$; pasión obsesiva: $\beta=0,15, t=3,82, p=0,000 ; \mathrm{F}_{(6,503)}=91,87, p=0,000 ; R^{2}=0,579$ ). Los resultados obtenidos demuestran que la pasión por el trabajo es un constructo teórica y empíricamente distinguible, capaz de explicar actitudes organizacionalmente relevantes.

Palabras clave: Pasión por el Trabajo, Flow, Implicación en el trabajo, Satisfacción Laboral.

\section{Work Passion: Evidence of Discriminant, Predictive and Incremental Validity}

\begin{abstract}
The aim of this study was to establish the discriminant, predictive and incremental validity of work passion regarding flow, job involvement and job satisfaction, respectively. An empirical-instrumental study was performed with a non-probabilistic sample (510 workers, $54 \%$ female). Exploratory structural equation modelling (ESEM) showed cross-saturation less than .30 and an adequate adjustment of an oblique hepta-factorial model (GFI $=0.92$, $\mathrm{CFI}=0.90$, TLI $=0.91$, RMSEA $=0.04)$. The average variance extracted, its square root and heterotrait-monotrait ratio (AVE $>0.50 ; \sqrt{ } \mathrm{AVE}<r$, HTMT $<0.90$ ) indicated discrimination capacity among all the constructs. Hierarchical multiple regression analysis confirmed the significant contribution of passion in explaining satisfaction (harmonious passion: $\beta=0.49, \quad t=10.96$, $p=0.000$; obsessive passion: $\beta=0.15, t=3.82, p=0.000 ; \mathrm{F}_{(6.503)}=91.87, p=0.000 ; R^{2}=0.579$ ). These results demonstrated work passion is a theoretically and empirically different construct, capable of explaining relevant organizational attitudes.
\end{abstract}

Keywords: Work Passion, Flow, Job Involvement, Job Satisfaction. 


\title{
Paixão Pelo Trabalho: Evidências de Validade Discriminante, Predictiva e Incremental
}

\begin{abstract}
Resumo: O objetivo deste estudo foi estabelecer a validade discriminante, preditiva e incremental da paixão pelo trabalho em relação ao flow, ao envolvimento eà satisfação no trabalho, respectivamente. Um estudo empírico-instrumental foi realizado com uma amostra não probabilística (510 trabalhadores, $54 \%$ mulheres). Análise de equações estruturais exploratórias (ESEM) mostraram saturação cruzada inferior a 0,30 e um ajuste adequado dum modelo hepta-fatorial oblíquo (GFI $=0,92, \mathrm{CFI}=0,90, \mathrm{TLI}=0,91, \mathrm{RMSEA}=0,04)$. Os valores da variância extraída média, da sua raiz quadrada e da proporção heterorasgo-monorasgo (AVE $>0,50$; $\sqrt{ }$ AVE $<r$, HTMT $<0,90$ ) indicaram capacidade discriminante entre os conceitos. Análises de regressão múltipla hierárquica confirmaram a contribuição significativa da paixão na explicação da satisfação (paixão harmoniosa: $\beta=0,49, t=10,96, p=0,000$; paixão obsessiva: $\beta=0,15, t=3,82, p=0,000 ; \mathrm{F}_{(6.503)}=91,87, p=0,000$; $\left.R^{2}=0,579\right)$. Os resultados demostraram que a paixão pelo trabalho é um constructo teórica e empiricamente diferente, capaz de explicar atitudes organizacionalmente relevantes.
\end{abstract}

Palavras-chave: Paixão pelo Trabalho, Flow, Envolvimento no Trabalho, Satisfação.

El advenimiento de la psicología organizacional positiva (Warren, Donaldson, \& Luthans, 2017) al escenario científico trajo consigo nuevos intereses académicos. El estudio del bienestar, de la resiliencia y del funcionamiento óptimo de las organizaciones y sus miembros comenzaron a ganar lugares destacados, opacando el protagonismo del que hasta entonces habían gozado el malestar y el conflicto. En este contexto, numerosas investigaciones se abocaron a examinar la influencia favorable de distintos estados y actitudes abiertas al cambio y al desarrollo. En el marco de esta renovada agenda, el estudio científico de la pasión alcanzó un lugar prominente, trasladándose desde una posición periférica al lugar central que ocupa en la literatura psicológica contemporánea.

La pasión por el trabajo se define como un estado de deseo persistente basado en valoraciones cognitivas y afectivas del propio trabajo (Perrewé, Hochwarter, Ferris, Mcallister, \& Harris, 2014). Se trata de un estado positivo y significativo de bienestar derivado de las evaluaciones que el sujeto efectúa de su situación laboral, y que resulta en un trabajo consistente y constructivo (Zigarmi, Nimon, Houson, Witt, \& Diehl, 2009). De acuerdo con los postulados del modelo dualista (Vallerand, 2015; Vallerand et al., 2003; Vallerand, \& Houlfort, 2003) y de la teoría de la autodeterminación (Ryan, \& Deci,
2016), el mecanismo explicativo que subyacente a la pasión es la internalización de la actividad en el self. No obstante, el modo como se lleva a cabo este proceso tiñe de connotaciones particulares a la pasión, haciendo posible distinguir dos variantes: la pasión armoniosa y la pasión obsesiva. En la pasión armoniosa, el trabajo ocupa un espacio significativo, pero no abrumador en la identidad, encontrándose en equilibrio con otras actividades. El individuo armoniosamente apasionado no experimenta un impulso incontrolable al realizar su trabajo, sino que éste es libremente elegido. Contrariamente, el individuo obsesivamente apasionado siente la necesidad imperiosa de trabajar, incluso, en momentos en que no debería. Este tipo de pasión puede llegar a acaparar un espacio desproporcionado en la identidad y, eventualmente, disminuir el interés en otras esferas vitales (Vallerand, \& Houlfort, 2003).

La pasión por el trabajo ha sido sindicada (Vallerand, \& Verner-Filion, 2013) como una candidata legítima para integrar la red nomológica de la psicología organizacional positiva. Sin embargo, aun cuando su validez de constructo ha sido ampliamente demostrada (Salesi, \& Omar, en prensa ${ }^{1}$; Marsch et al., 2013; Orgambídez-Ramosa, Borrego-Alés, \& Gonçalvesa, 2014; Vallerand et al., 2003; Vallerand, \& Houlfort, 2003; Zito \& Colombo, 2017), su semejanza

\footnotetext{
${ }^{1}$ Salessi, S. \& Omar, A. (en prensa). Validación Escala Pasión por el trabajo: Un estudio instrumental con trabajadores argentinos. Psykhé.
} 
con otros conceptos amerita un examen más profundo. Entre los constructos afines, el engagementy la adicción al trabajo han sido los primeros en acaparar el interés de los especialistas. La investigación al respecto señala que se trata de constructos empírica y conceptualmente distinguibles (Birkeland, \& Buch, 2015; Gorgievski, \& Bakker, 2010). En este sentido, por ejemplo, el engagement constituye un estado relativamente transitorio que oscila diariamente al ritmo de las experiencias laborales (Engeser, \& Baumann, 2016). Contrariamente, la pasión se encuentra exenta del influjo de los pormenores cotidianos (Vallerand, \& Houlfort, 2003). Paralelamente, la adicción es incompatible con experiencias de placer y disfrute (Taris, Van Beek, \& Schaufeli, 2014); mientras que, la pasión (incluso obsesiva) supone experiencias de afecto positivo relacionadas con el trabajo (Orgambídez-Ramosa et al., 2014; Salesi, \& Omar, en prensa²; Zito, \& Colombo, 2017).

Además de estos constructos, se ha advertido (Vallerand, 2015) que otros conceptos que integran el entramado nomológico de la psicología positiva también podrían ser objeto de controversia. Entre éstos, el flow relacionado con el trabajo (Bakker, 2008) y la implicación laboral (Kanungo, 1982) reclaman especial atención. No obstante, hasta el momento ningún estudio se ha dedicado a examinar las diferencias entre estos constructos, por lo que una investigación focalizada en analizar su validez discriminante realizaría un aporte sustancial a la literatura organizacional. Del mismo modo, las investigaciones sobre el impacto del flow, de la implicación y de la pasión sobre el comportamiento de los trabajadores y sobre los resultados organizacionales han discurrido por carriles paralelos, asiladas unas de otras. Como corolario, ninguno de los estudios hasta ahora desarrollados se ha abocado a determinar el aporte específico y la importancia relativa de cada constructo en la explicación de resultados organizacionalmente relevantes, tales como, por ejemplo, la satisfacción laboral. Frente a este panorama, el presente estudio se propuso un objetivo doble: (a) examinar la validez discriminante de la pasión por el trabajo respecto a los constructos de flow e implicación laboral y; (b) determinar su validez predictiva e incremental (más allá del flow y de la implicación con el trabajo) en la explicación de la satisfacción laboral.

\section{Validez discriminante, predictiva e incremental en la investigación psicológica}

En los últimos años, el análisis de la validez discriminante, predictiva e incremental se ha tornado un tópico crucial en la investigación psicológica en general y, organizacional en particular. Este renovado interés posiblemente se base en la popularidad de la que gozan en estas disciplinas los métodos basados en la estructura de covarianzas tales como, por ejemplo, el enfoque SEM (Structural Equation Modelling). Este método utiliza compuestos lineales de los ítems como indicadores observables de los factores latentes con miras a estimar las relaciones entre ellos. Por ende, es indispensable que los modelos de medición "reflejen" (Henseler, Ringle, \& Sarstedt, 2015) lo que pretenden medir. De aquí que la validez discriminante se imponga como un preexisto inexorable para cualquier análisis que pretenda examinar las relaciones causales de tipo lineal sobre datos no experimentales.

Asimismo, determinar el grado en el que una variable latente explica y mejora la predicción de un criterio en el contexto de otros predictores, también constituye otro aspecto sustancial en la investigación psicológica. Los beneficios de la simple combinación de medidas no deben ser asumidos por defecto, sino que deben ser demostrados empíricamente. A su vez, la utilización de diferentes variables latentes en un modelo explicativo ha de ser contemplada desde la perspectiva de la validez predictiva e incremental; vale decir, estableciendo en qué grado su inclusión proporciona una mejora sustancial en el ajuste del modelo (Husley, \& Meyer, 2003; Sackett, Dahlke, Shewach, \& Kuncel, 2017).

Establecer la validez conlleva una serie de beneficios prácticos. Por un lado, la validez discriminante demuestra que la medición de un constructo es empíricamente única, representando a un fenómeno en una forma que otros constructos del mismo modelo estructural no capturan (Farrell, 2010). Por otro lado, la validez predictiva e incremental posibilitan estimar cuantitativamente el grado en que un constructo incrementa la capacidad explicativa, y determinar si esta varianza única es significativa. En última instancia, permiten determinar el grado en el que la inclusión de diversas medidas/variables en el proceso de evaluación mejora consistentemente el poder predictivo y, en consecuencia, la toma de decisiones (Merino, 2014; Sackett et al., 2017).

${ }^{2}$ Salessi, S. \& Omar, A. (en prensa). Validación Escala Pasión por el trabajo: Un estudio instrumental con trabajadores argentinos. Psykhé. 


\section{Pasión por el trabajo, flow e implicación laboral como constructos distinguibles}

Para ser considerados distintos, dos constructos deben ser distinguibles tanto conceptual como empíricamente. En lo que hace al primer requisito, se ha objetado (Le, Schmidt, Harter, \& Lauver, 2010) que es algo débil y, en general, puede satisfacerse fácilmente. En efecto, la suposición implícita que subyace a este criterio es que, si los investigadores pueden hacer una distinción lógica entre los constructos, esta distinción también existirá en la "mente" de los participantes del estudio. Sin embargo, esta aseveración puede no ser válida (Harter, \& Schmidt, 2008). El segundo requerimiento, en tanto, sostiene que dos constructos supuestamente distintos no deben correlacionar entre sí de modo "perfecto", una vez que los efectos producidos por los sesgos de la medición son controlados. Si las correlaciones entre dos constructos son próximas a 1 , tales constructos son indiferenciables; vale decir, empíricamente redundantes (Le et al., 2010; Morrow, 1983; Rousseau, 2007; Schwab, 1980).

En lo que hace a los constructos bajo análisis, es posible plantear una distinción teórica que permita satisfacer el primer requisito de validez discriminante. En este sentido, el flow ha sido definido como una experiencia óptima a corto plazo caracterizada por la inmersión absoluta en el trabajo, las emociones positivas y la motivación intrínseca frente a la tarea (Bakker, 2008). Si bien, al igual que la pasión, presupone experiencias de placer y motivación, el flow constituye un fenómeno temporal, circunscripto al tiempo que insuma la realización de la tarea (Bakker, 2008; Engeser, \& Baumann, 2016). Contrariamente, la pasión constituye un estado psicológico de deseo por el trabajo que persiste en el tiempo (Vallernad et al., 2003; Vallerand, \& Houlfort, 2003). Del mismo modo, la implicación laboral hace referencia al grado en que una persona está cognitivamente involucrada con su trabajo actual, caracterizándose como un estado positivo de compromiso, identificación y participación en el trabajo (Kanungo, 1982). Aun cuando en la implicación el trabajo ocupa un lugar prominente en la vida de la persona, no necesariamente se erige como un componente de su identidad. En efecto, la implicación puede describirse como una creencia descriptiva determinada situacionalmente, ligada a la percepción del potencial de un trabajo particular para satisfacer las necesidades más salientes del individuo (Kanungo, 1982). La pasión, en cambio, consiste en una fuerte inclinación hacia un trabajo que genera placer y que se considera central en la vida, siendo su nota distintiva la internalización del mismo en el self. De aquí que, en la pasión, el trabajo constituya una parte sustancial de la identidad (Ryan, \& Deci, 2016). Con base en estos antecedentes teóricos se hipotetiza que:

Hipótesis 1: la pasión por el trabajo (armoniosa y obsesiva) será empíricamente distinguible del low y de la implicación laboral.

\section{Pasión por el trabajo, flow e implicación como predictores de satisfacción laboral}

En lo que respecta a la validez predictiva de las variables aquí analizadas, la evidencia publicada demuestra que, tanto el flow como la implicación laboral conllevan beneficios para las organizaciones y para los propios trabajadores. En este sentido, contar con empleados consustanciados con sus trabajos supone para las organizaciones disponer de miembros emocionalmente más comprometidos, dedicados, perseverantes, dispuestos a realizar esfuerzos extraordinarios para alcanzar los objetivos e incrementar su desempeño (Johari, \& Yahya, 2016; Salesi, \& Omar, en prensa ${ }^{\mathbb{D}}$ ). Para los empleados, en tanto, la implicación con el trabajo conduce a mayores niveles de bienestar subjetivo, satisfacción y enriquecimiento entre las esferas del trabajo y la familia (Bayraktar, Araci, Karacay, \& Calisir, 2017; Cheng, 2014; Konradt, \& Garbers, 2016). Del mismo modo, contar con trabajadores inmersos en su tarea, intrínsecamente interesados y capaces de obtener placer de su trabajo allana el camino a resultados organizacionales destacados (Bakker, 2008). Al mismo tiempo, altos niveles de flow conducen a mayor bienestar entre los empleados, emociones positivas, engagement y satisfacción (llies et al., 2017; Maeran, \& Cangiano, 2013). Frente a estos hallazgos se conjetura que:

Hipótesis 2: el flow y la implicación en el trabajo predecirán positivamente a la satisfacción laboral.

Paralelamente, la literatura especializada indica que la pasión por el trabajo se asocia a un amplio abanico de conductas positivas. Así, por ejemplo, si bien no se traduce directamente en rendimientos más eficientes, sí se ha comprobado que se relaciona a mayores niveles de concentración y el engagement, redundando así en desempeños laborales destacados (Astakhova, \& Porter, 2015). A su vez, se ha confirmado que la pasión predice positivamente el enriquecimiento trabajo-familia, el compromiso y la identificación con la organización (Curran, Hill, Appleton, Vallerand, \& Standage, 
2015). Sumado a eso, diversos estudios (Birkeland, \& Buch, 2015; Houlfort, Philippe, Vallerand, \& Ménard, 2014) han comprobado que la pasión realiza un aporte sustancial en la explicación del burnout, del bienestar subjetivo y de la satisfacción con la vida. Con base en estos referentes empíricos se presupone que:

Hipótesis 3: la pasión por el trabajo predecirá significativamente (más allá del flowy de la implicación) a la satisfacción laboral.

\section{Método}

\section{Diseño}

Se realizó un estudio de corte transversal, descriptivo-correlacional, encuadrado en la categoría de estudios instrumentales (Ato, López, \& Benavente, 2013).

\section{Participantes}

Se integró una muestra inicial de 537 participantes, de los cuales fueron descartados 16 casos incompletos. La muestra definitiva quedó conformada por 521 trabajadores ( $54 \%$ mujeres) de diversas organizaciones argentinas públicas y privadas localizadas en el centro-sur de la provincia de Santa Fe y norte de la provincia de Buenos Aires. El promedio de edad fue de 32 años $(D T=12,24)$. La antigüedad laboral media fue de 5 años $(D T=10,21)$. El $74.5 \%$ de la muestra tenía estudios superiores terciarios y/o universitarios. El 72\% de los trabajadores tenía un trabajo efectivo, en tanto que el $28 \%$ un contrato de duración limitada. En promedio, los empleados trabajaban 42,5 horas por semana $(D T=6,50)$. El $58,7 \%$ trabajaba en el sector privado. La distribución por rubros de actividad fue la siguiente: $34,2 \%$ comercio; $28,5 \%$ industria; $22,6 \%$ salud y; $14,7 \%$ educación.

\section{Procedimiento}

La recolección de los datos se llevó a cabo en el seno de las organizaciones que aceptaron participar de la investigación, en días y horarios previamente pautados con las autoridades, y en los lugares físicos que las mismas dispusieron a tal efecto. La participación de los trabajadores fue voluntaria, anónima y confidencial. No se brindaron incentivos de ningún tipo. La administración de las técnicas estuvo a cargo de personal entrenado a tal efecto. En todos los casos, los participantes completaron de manera individual un cuadernillo conteniendo en su primera hoja el objetivo del estudio y las instrucciones para responder; en la segunda hoja el formulario de consentimiento informado y; en las restantes páginas los ítems correspondientes a los instrumentos que a continuación se describen, distribuidos aleatoriamente. La ejecución del estudio se llevó a cabo en un todo de acuerdo con los lineamientos éticos establecidos por la Asociación Americana de Psicología y las recomendaciones del CONICET para las investigaciones en las ciencias sociales y humanas (Resolución $n^{\circ}$ 2.827/06).

\section{Instrumentos}

Escala de pasión por el trabajo. Se aplicó la adaptación argentina (Salesi, \& Omar, en prensa ${ }^{1}$ ) de la escala de Vallerand et al. (2003). El instrumento se encuentra integrado por 14 ítems con formato Likert de 5 puntos (variando desde $1=$ Totalmente en desacuerdo $5=$ Totalmente de acuerdo) distribuidos proporcionalmente entre las sub-escalas de pasión armónica (ej.: "las cosas que aprendo en este trabajo hacen que lo valore más todavía", $\alpha=0,79$ ) y pasión obsesiva (ej.: "el impulso es tan fuerte que no puedo dejar de hacer este trabajo", $\alpha=0,80$ ).

Escala de implicación en el trabajo. Se empleó la versión adaptada (Salesi, \& Omar, en prensa²) de la escala desarrollada por Kanungo (1982). El instrumento está integrado por 9 ítems (ej.: "mi trabajo ocupa un lugar irremplazable en mi vida"; $\alpha=0,89$ ) valorados sobre una escala tipo Likert de 5 puntos ( $1=$ Totalmente en desacuerdo; $5=$ Totalmente de acuerdo).

Escala de flow relacionado con el trabajo. Se aplicó la adaptación argentina (Autor \& Autor, en prensa ${ }^{c}$ ) de la escala elaborada por Bakker (2008). El instrumento está integrado por 12 ítems distribuidos en tres sub-escalas: absorción (4 ítems; ej.: "cuando estoy trabajando no pienso en otra cosa”; $\alpha=0,80$ ); disfrute ( 4 ítems; ej.: "cuando estoy trabajando me siento feliz"; $\alpha=0,81$ ), y motivación intrínseca (4 ítems; ej.: "mi trabajo me motiva por sí mismo y no por las recompensas que recibo"; $\alpha=0,78)$. Los mismos son valorados sobre una escala tipo Likert de 5 puntos $(1=$ Totalmente en desacuerdo; 5 = Totalmente de acuerdo).

Escala de satisfacción laboral. Se utilizó la Escala de Satisfacción Laboral Genérica (Salesi, \& Omar, 2016). El instrumento está integrado por 7 ítems (ej.: "en mi trabajo puedo aplicar todas mis habilidades" $\alpha=0,87$ ) valorados sobre una escala tipo Likert de 5 puntos $(1=$ Totalmente en desacuerdo; 5 = Totalmente de acuerdo). 


\section{Estrategia de análisis de datos}

Para el procesamiento de los datos se utilizaron los programas SPSS (versión 23.0) y Mplus (versión 7.3; Muthén, \& Muthén, 2014) y G*Power. La estrategia implementada para el análisis se desarrolla a continuación.

Análisis exploratorios preliminares. Se examinó la matriz de datos con miras a detectar valores perdidos y/o extremos. Se identificaron los casos atípicos mediante el cálculo de puntuaciones $Z$ y las distancias de Mahalanobis al cuadrado $\left(D^{2}\right)$ para cada variable. Se consideraron atípicas univariadas aquellas observaciones alejadas más de 3,5 DT de la media, y atípicas multivariadas a aquellas que presentasen $D^{2}$ con probabilidad igual o inferior a 0,001 (Hair, Black, Babin, Anderson, \& Tatham, 2010; Tabachnick, \& Fidell, 2013). Se realizó un análisis de los ítems mediante el cálculo de estadísticos descriptivos (medías y desviaciones típicas), coeficientes de asimetría y curtosis e índices de discriminación (a partir del cómputo de las correlaciones ítem-total corregidas). Valores de asimetría y curtosis inferiores a $+2,00$ y correlaciones positivas superiores a 0,30 se consideraron adecuadas (Tabachnick, \& Fidell, 2013). Por último, se examinó el cumplimiento de los supuestos de independencia entre los residuos (mediante el estadístico $d$ de Durbin-Watson), homocedasticidad, linealidad, normalidad (a través de la inspección de los diagramas de dispersión de los Pronósticos Tipificados, de los Residuos Tipificados, del histograma de los residuos tipificados, del gráfico de probabilidad normal y del diagrama de regresión parcial), y libre colinealidad (mediante el índice de tolerancia y su inverso FIV). Se consideraron óptimos valores del estadístico $d$ comprendidos entre 1,5 y 2,5; valores superiores a 0,10 en el índice de tolerancia y valores inferiores a 3 en su inverso FIV (Tabachnick, \& Fidell, 2013; Hair et al., 2010).

Análisis de ecuaciones estructurales exploratorio. Con miras a determinar si los ítems "reflejan" adecuadamente los constructos que pretenden medir se realizó un análisis de ecuaciones estructurales exploratorio (ESEM, Exploratory Structural Equation Modelling). Este método genera estimaciones más precisas de las cargas factoriales y las correlaciones entre factores, al permitir la estimación libre de las cargas cruzadas. Por tanto, es menos restrictivo que el análisis factorial confirmatorio tradicional y se ajusta más a la realidad de las medidas en Psicología, donde habitualmente los ítems no constituyen indicadores "perfectos" del constructo, presentando saturaciones menores (aunque no nulas) en los otros factores (Marsh, Morin, Parker, \& Kaur, 2014). Para la estimación del modelo se empleó el método de mínimos cuadrados no ponderados con ajuste en medias y varianzas (WLSMV, Weighted Least Squares Means and Variance Adjusted). Asimismo, se utilizó rotación oblicua target, ya que proporciona la solución rotada más próxima a una configuración pre-especificada de cargas, permitiendo así utilizar el ESEM en un modo confirmatorio. Para evaluar la bondad de ajuste se analizó que: (a) el índice de bondad de ajuste (GFI, Goodness of Fit Índex), el índice de ajuste comparativo (CFI, Comparative Fit Índex) y el índice de Tucker-Lewis (TLI, Tucker-Lewis Índex) alcanzaran valores iguales o superiores a 0,90; y (b) que el error cuadrático medio de aproximación (RMSEA, Root Mean Square Error of Approximation) fuera inferior a 0,08 (Muthén, \& Muthén, 2014).

Validez discriminante. Se obtuvo evidencia de validez discriminante mediante tres procedimientos: (a) el cálculo de la varianza media extraída (AVE, Average Variance Extracted) y de su raíz cuadrada, respectivamente; (b) el cómputo de la proporción heterorasgo-monorasgo (HTMT, Heterotrait-Monotrait Ratio) y; (c) el cálculo de las correlaciones entre los constructos estudiados. El AVE permite estimar la varianza común entre los indicadores y su factor latente, considerando apropiados valores iguales o superiores a 0,50 . Asimismo, valores de su raíz cuadrada superiores a la correlación entre los factores latentes, se asume como evidencia de que cada constructo comparte más varianza con sus indicadores que con los demás (Bagozzi, \& Yi, 2012). La proporción HTMT, en tanto, es la media de las correlaciones entre los indicadores que miden diferentes constructos (correlación HT, Heterotrait-Heteromethod), en relación con el promedio de las correlaciones de indicadores dentro del mismo constructo (correlaciones MT, Monotrait-Heteromethod). Valores inferiores a 0,90 demuestran una adecuada validez discriminante (Henseler et al., 2015).

Validez predictiva e incremental. Para evaluar la validez predictiva e incremental de la pasión se adoptó la estrategia sugerida por Hunsley y Meyer (2003). En este sentido, se realizó un análisis de regresión múltiple jerárquica utilizando el método introducir. Este procedimiento permite aislar la varianza específica de satisfacción laboral (variable criterio) explicada por las variables de interés una vez controlado el efecto de las restantes. En un primer modelo se ingresaron las tres dimensiones del flow y la impli- 
cación laboral. En un segundo modelo se añadieron la pasión armónica y la pasión obsesiva. Las medidas de ajuste analizadas fueron el $R^{2}$ y el Criterio de Información de Akaike (AIC). Se estableció el tamaño del efecto mediante el cómputo del coeficiente $f^{2}$ de Cohen, considerando moderado y grande valores de 0,15 y 0,35 , respectivamente. La utilidad pragmática del cambio en el $R^{2}$ fue determinada con base en la correlación semiparcial $\left(\mathrm{r}_{\mathrm{sp}}\right)$, que permite conocer el grado de correlación entre el criterio y cada variable predictora mientras se controlan los efectos de las demás variables independientes del modelo. Siguiendo a Husley y Meyer (2003), una correlación semiparcial del orden de 0,15 a 0,20 indica una contribución razonable a la ecuación. Adicionalmente, se llevó a cabo un análisis de la comunalidad. El mismo permite dividir el porcentaje de varianza explicada total en tantos porcentajes de varianza específica como predictores analizados, y en un porcentaje de varianza común entre todos ellos. Para obtener las varianzas únicas se computó el cuadrado de las correlaciones semiparciales. Para obtener la varianza común se restó la varianza específica de cada uno de los predictores a la varianza total explicada (Reichwein-Zientek, \& Thompson, 2006).

\section{Resultados}

\section{Análisis exploratorios}

El porcentaje de datos perdidos en cada ítem no superó el $5 \%$. Los valores faltantes fueron reemplazados mediante el método de Estimación-Maximización (Tabachnick, \& Fidell, 2013). No se registraron problemas de asimetría y curtosis, quedando dichos valores comprendidos entre los límites establecidos [Flow: asimetría $=-1,08$ a $0,92(\mathrm{Mdn}=0,52)$, curtosis $=-1,31$ y 1,05 $(\mathrm{Mdn}=0,22)$; Implicación Laboral: asimetría $=-1,28$ a $0,93(\mathrm{Mdn}=-0,25)$, curtosis $=-1,11$ a 1,07 (Mdn $=0,35)$; Satisfacción Laboral: asimetría $=-1,15$ y $1,27(\mathrm{Mdn}=0,78)$, curtosis $=-1,12$ y 0,91 (Mdn = 0,74); Pasión Armoniosa: asimetría = -0,97 y $1.03(\mathrm{Mdn}=-0,89)$, curtosis $=-1,28$ y 0,96 $(\mathrm{Mdn}$ = 0,74); Pasión Obsesiva: asimetría $=-1,26$ y 0,99 $(\operatorname{Mdn}=-0,89)$, curtosis $=-1,17$ y $1,02(\operatorname{Mdn}=-0,35)]$. Las correlaciones ítem-total fueron todas positivas, variando entre 0,46 y 0,72 . Se detectaron 11 casos atípicos, 6 de los cuales satisfacían la condición para considerarse atípicos multivariados (Hair et al.,
2010; Tabachnick, \& Fidell, 2013). Los mismos fueron eliminados, quedando la muestra final constituida por 510 participantes; tamaño adecuado para la ejecución de los análisis previstos (Lloret, Ferreres, Hernández, \& Tomás, 2017). Los gráficos indicaron el cumplimiento de los supuestos de homocedasticidad, linealidad y normalidad. Se obtuvieron valores de tolerancia entre 0,63 y 0,94 , y entre 1,03 y 1,15 en su inverso FIV. El estadístico $d$ fue de 1,74. Tales resultados mostraron un adecuado cumplimiento de los supuestos multivariados (Hair et al., 2010; Tabachnick \& Fidell, 2013).

\section{Análisis de ecuaciones estructurales exploratorio}

La matriz de datos fue considerada factorizable, habida cuenta que tanto el test de esfericidad de Bartlett $\left(\chi_{(98,510)}^{2}=11286,49, p=0,000\right)$, como el índice de adecuación muestral de Kaiser-Meyer-Olki $(0,92)$ mostraron valores apropiados (Lloret et al., 2017). Los índices obtenidos (GFI $=0,92, \mathrm{CFI}=0,90$, TLI $=0,91$, RMSEA $=0,04$, IC95\% $[0,03,0,05])$ para el modelo de medida hepta-factorial (siete factores correlacionados: pasión armoniosa, pasión obsesiva, implicación con el trabajo, absorción, disfrute, motivación intrínseca y satisfacción laboral) indicaron un adecuado ajuste (Muthén, \& Muthén, 2014). En todos los casos los ítems pesaron más en el factor latente correspondiente. Un análisis más pormenorizado muestra que el ítem 8 ("no puedo vivir sin este trabajo") y el ítem 14 ("no puedo imaginarme sin hacer este trabajo"), correspondientes a la escala de pasión obsesiva, presentaron las saturaciones cruzadas más elevadas en el factor 3 (implicación laboral). A su vez, los ítems 20 ("vivo, como y respiro por mi trabajo"), 22 ("mi trabajo ocupa un lugar central en mi vida") y 23 ("me gustaría estar trabajando la mayor parte del tiempo") pertenecientes a la escala de implicación, mostraron saturaciones cruzadas más altas en los factores de pasión armoniosa y obsesiva, siendo más elevadas particularmente en este último. Finalmente, el ítem 23 ("me gustaría estar trabajando la mayor parte del tiempo") de la escala implicación, y el ítem 33 ("quiero trabajar incluso en mi tiempo libre) correspondiente a la dimensión motivación intrínseca de la escala flow, presentaron sus mayores saturaciones cruzadas en los factores de pasión obsesiva e implicación laboral. No obstante, en ninguno de los casos analizados las cargas fueron superiores 
Tabla 1

Saturaciones cruzadas correspondientes a los ítems de pasión por el trabajo, implicación, flow y satisfacción laboral.

\begin{tabular}{|c|c|c|c|c|c|c|c|}
\hline Contenido del item & I & II & III & IV & $\mathrm{V}$ & $\mathrm{VI}$ & VII \\
\hline 1. Este trabajo me permite vivir diversas experiencias & 0,78 & 0,02 & 0,01 & 0,02 & $-0,05$ & 0,01 & 0,03 \\
\hline 2. Las cosas que aprendo en este trabajo hacen que lo valore más & 0,71 & $-0,07$ & $-0,10$ & $-0,06$ & 0,12 & 0,01 & 0,06 \\
\hline 3. Este trabajo está en equilibrio con otras actividades de mi vida & 0,65 & $-0,02$ & $-0,04$ & $-0,11$ & $-0,01$ & 0,03 & 0,09 \\
\hline 4. Este trabajo refleja mis cualidades & 0,78 & 0,09 & 0,22 & $-0,09$ & $-0,07$ & $-0,05$ & 0,12 \\
\hline 5. Estoy completamente comprometido con este trabajo & 0,62 & 0,05 & 0,13 & 0,04 & $-0,04$ & 0,04 & 0,05 \\
\hline 6. En este trabajo vivo momentos inolvidables & 0,73 & $-0,02$ & $-0,07$ & 0,05 & $-0,01$ & $-0,05$ & 0,06 \\
\hline 7. Este trabajo es una pasión que todavía logro controlar & 0,52 & $-0,06$ & $-0,18$ & 0,08 & 0,07 & 0,07 & 0,01 \\
\hline 8. No puedo vivir sin este trabajo & 0,09 & 0,70 & 0,21 & 0,01 & $-0,03$ & 0,05 & 0,01 \\
\hline 9. Dependo emocionalmente de este trabajo & 0,03 & 0,75 & $-0,05$ & $-0,07$ & 0,02 & $-0,01$ & 0,02 \\
\hline 10. Me cuesta controlar la necesidad de realizar este trabajo & 0,09 & 0,81 & $-0,06$ & 0,01 & 0,15 & $-0,15$ & 0,04 \\
\hline 11. El impulso es tan fuerte, que no puedo dejar de hacer este trabajo & 0,04 & 0,58 & $-0,14$ & 0,02 & $-0,01$ & 0,01 & 0,01 \\
\hline 12. Tengo un sentimiento casi obsesivo por este trabajo & 0,02 & 0,74 & 0,20 & $-0,04$ & $-0,10$ & $-0,03$ & 0,02 \\
\hline 13. Mi estado de ánimo depende de este trabajo & 0,07 & 0,65 & 0,01 & $-0,11$ & 0,13 & 0,05 & 0,01 \\
\hline 14. No puedo imaginarme sin hacer este trabajo & 0,08 & 0,75 & 0,20 & $-0,13$ & 0,02 & $-0,07$ & 0,03 \\
\hline 15. Estoy totalmente comprometido con mi trabajo & 0,16 & 0,18 & 0,3 & 0,09 & 0,03 & 0,09 & 0,05 \\
\hline 16. Las cosas más importantes que vivo tienen que ver con & 0,05 & 0,07 & 0,84 & 0,04 & 0,14 & $-0,10$ & 0,10 \\
\hline 17. Mi trabajo es sólo una pequeña parte de mí (*) & 0,03 & $-0,13$ & 0,85 & $-0,13$ & 0,22 & 0,04 & 0,12 \\
\hline 18. Tengo una conexión con mi trabajo muy difícil de romper & 0,09 & 0,12 & 0,71 & 0,06 & $-0,01$ & 0,08 & 0,03 \\
\hline 19. La mayoría de mis objetivos están relacionados a mi trabajo & $-0,10$ & 0,16 & 0,72 & $-0,07$ & $-0,10$ & $-0,01$ & 0,10 \\
\hline 20. Vivo, como y respiro por mi trabajo & 0,06 & $-0,25$ & 0,87 & $-0,09$ & 0,02 & $-0,04$ & -05 \\
\hline 21. A menudo me siento "desenganchado" de mi & $-0,03$ & $-0,08$ & 0,82 & 0,08 & 0,11 & 0,05 & 0,15 \\
\hline 22. Mi trabajo ocupa un lugar central en mi vida & 0,17 & 0,24 & 0,79 & $-0,01$ & 0,08 & 0,08 & 0,03 \\
\hline 23. Me gustaría estar trabajando la mayor parte del tiempo & 0,12 & 0,23 & 0,63 & 0,07 & $-0,09$ & 0,21 & 0,11 \\
\hline 24. Cuando estoy trabajando no pienso en otra cosa & 0,19 & 0,17 & 0,01 & 0,77 & 0,07 & $-0,04$ & 0,01 \\
\hline 25. Me dejo "llevar" por mi trabajo & 0,04 & 0,06 & $-0,01$ & 0,61 & 0,01 & 0,11 & 0,02 \\
\hline 26. Cuando trabajo me olvido de todo lo que me rodea & 0,05 & 0,03 & 0,10 & 0,80 & 0,05 & 0,13 & 0,09 \\
\hline 27. Estoy muy concentrado en mi trabajo & 0,01 & $-0,01$ & 0,02 & 0,72 & 0,03 & 0,05 & 0,01 \\
\hline 28. Mi trabajo me produce lindas sensaciones & $-0,18$ & 0,07 & 0,11 & 0,11 & 0,52 & 0,08 & 0,19 \\
\hline 29. Cuando estoy trabajando me siento feliz & $-0,19$ & 0,01 & 0,08 & $-0,14$ & 0,53 & 0,10 & 0,18 \\
\hline 30. Hago mi trabajo con mucho entusiasmo & 0,06 & $-0,07$ & 0,20 & 0,09 & 0,62 & 0,13 & 0,16 \\
\hline 31. Me gusta mi trabajo & 0,15 & 0,05 & 0,17 & 0,01 & 0,19 & 0,77 & 0,15 \\
\hline 32. Mi trabajo me interesa por sí mismo y no p & 0,03 & 0,02 & 0,18 & 0,03 & 0,09 & 0,64 & 0,04 \\
\hline 33. Quiero trabajar incluso en mi tiempo libre & 0,01 & 0,20 & 0,24 & $-0,06$ & 0,03 & 0,58 & 0,02 \\
\hline 34. Haría este trabajo, aunque no me pagaran por él & 0,03 & 0,07 & 0,09 & $-0,07$ & 0,10 & 0,62 & 0,09 \\
\hline 35. En mi trabajo puedo aplicar mis habilidades & 0,13 & $-0,03$ & 0,03 & $-0,01$ & 0,04 & $-0,08$ & 0,57 \\
\hline 36. En mi trabajo recibo reconocimiento por mi buen desempeño & 0,10 & 0,09 & 0,05 & $-0,07$ & $-0,03$ & 0,02 & 0,54 \\
\hline 37. Me siento bien trabajando para esta empresa & $-0,01$ & $-0,01$ & $-0,01$ & 0,03 & $-0,04$ & 0,03 & 0,65 \\
\hline 38. Me siento a gusto con mis compañeros de trabajo & 0,02 & -001 & 0,02 & $-0,01$ & 0,01 & 0,01 & 0,74 \\
\hline 39. Mi trabajo me da seguridad laboral & 0,13 & 0,12 & 0,01 & 0,01 & 0,04 & $-0,04$ & 0,60 \\
\hline 40. Mi salario es apropiado & $-0,01$ & $-0,02$ & 0,01 & $-0,01$ & $-0,02$ & 0,03 & 0,68 \\
\hline 41. Considerándolo en términos generales tengo un buen trabajo & 0,13 & 0,09 & $-0,07$ & 0,08 & $-0,14$ & $-0,07$ & 0,80 \\
\hline
\end{tabular}

Nota: I (pasión armoniosa); II (pasión obsesiva); III (implicación en el trabajo); IV (absorción);V (disfrute); VI (motivación intrínseca); VIII (satisfacción laboral). El asterisco indica los ítems inversos. En negrita se indican las saturaciones en el factor correspondiente. 
a .30 (Lloret et al., 2017; Marsch et al., 2014; Muthén, \& Muthén, 2014), proporcionando evidencia preliminar favorable a la validez discriminante. La Tabla 1 informa las saturaciones de los ítems en cada factor.

\section{Análisis de validez discriminante}

La Tabla 2 muestra los coeficientes de correlación, los valores correspondientes al índice AVE y a su raíz cuadrada, y la proporción HTMT.

De los resultados se desprende que, tanto la pasión armoniosa como obsesiva mostraron relaciones positivas con la implicación, la satisfacción laboral y el flow relacionado con el trabajo. No obstante, en el caso de la pasión obsesiva las correlaciones con las dimensiones de disfrute y motivación intrínseca, así como con la satisfacción laboral fueron inferiores. Las raíces cuadradas del AVE correspondiente a la pasión armoniosa y obsesiva fueron mayores que la correlación entre éstos y los restantes constructos estudiados. Del mismo modo, el valor del AVE se ubicó por encima del mínimo recomendado, y en todos los casos la proporción HTMT fue inferior a 0,90 (Bagozzi \& Yi, 2012; Henseler et al., 2015). En conjunto, estos resultados proporcionan apoyo empírico a la hipótesis 1, que postulaba que la pasión por el trabajo sería un constructo empíricamente distinguible del flow y de la implicación laboral.

\section{Análisis de validez predictiva e incremental}

El análisis de regresión realizado muestra que, en el primer modelo, las dimensiones de disfrute, motivación intrínseca, absorción e implicación laboral realizaron una contribución significativa a la satisfacción, explicando en conjunto el $53.1 \%$ de su varianza $\left(\mathrm{F}_{(4,505)}=\right.$ $113,37, p=0,000$ ). Tales resultados confirman empíricamente la hipótesis 2, que adelantaba el rol predictor del flowy de la implicación laboral. Al ingresar la pasión armoniosa y la pasión obsesiva en el segundo modelo se registró un aumento del $4,8 \%$ en la varianza explicada del criterio $\left(\mathrm{F}_{6,503)}=91,87, p=0,000\right)$. Este incremento fue superior al mínimo de varianza única sugerido (Hunsley, \& Meyer, 2003) y estadísticamente significativo. La Tabla 3 informa los coeficientes de regresión estandarizados y los estadísticos de ajuste de cada modelo contrastado.

Los valores de $R^{2}$ y del AIC muestran que la varianza explicada por el modelo que incluye a la pasión por el trabajo aporta mejor información predictiva de la satisfacción laboral. La inspección de los coeficientes de regresión permite señalar que es la pasión armoniosa la que realiza una contribución más elevada, aunque ambas predicen positivamente a la satisfacción. El coeficiente $f$ obtenido para el $R^{2}$ correspondiente al segundo modelo demostró que se trata de un efecto grande y significativo. Estos resultados proporcionan apoyo empírico a las hipótesis 3, desde el momento que, más allá del aporte explicativo correspondiente a la implicación y al flow, la pasión por el trabajo contribuye significativa e incrementalmente a la predicción de la satisfacción laboral. En la Figura se ilustra gráficamente la varianza común y las varianzas específicas correspondientes (Reichwein-Zientek, \& Thompson, 2006).

\section{Discusión}

En los últimos años, diversos especialistas (Le et al., 2010; Morrow, 1983; Rousseau, 2007; Schwab, 1980) vienen advirtiendo sobre la necesidad de analizar la redundancia entre constructos, con miras a evitar la proliferación indiscriminada de conceptos y garantizar la parsimonia en la investigación organizacional. Atendiendo a este llamado, el presente estudio se propuso, por un lado, dem-

Tabla 2

Estadísticos descriptivos, índices de correlación, varianza media extraída y proporción heterorasgo-monorasgo de las variables latentes bajo estudio.

\begin{tabular}{lccccccccccc}
\hline & $\mathrm{M}$ & $\mathrm{DT}$ & 1 & 2 & 3 & 4 & 5 & 6 & 7 & AVE & $\sqrt{\text { AVE }}$ \\
\hline 1. Pasión armoniosa & 3,28 & 1,29 & - & .41 & 0,61 & 0,50 & 0,64 & 0,62 & 0,56 & 0,77 & 0,87 \\
2. Pasión obsesiva & 2,30 & 1,32 & 0,48 & - & 0,68 & 0,47 & 0,28 & 0,48 & 25 & 0,80 & 0,89 \\
3. Implicación con el trabajo & 2,95 & 1,46 & 0,71 & 0,77 & - & 0,59 & 0,55 & 0,62 & 0,58 & 0,73 & 0,85 \\
4. Absorción & 3,16 & 1,21 & 0,67 & 0,51 & 0,65 & - & 0,41 & 0,45 & 0,42 & 0,76 & 0,87 \\
5. Disfrute & 3,48 & 1,39 & 0,72 & 0,33 & 0,59 & 0,47 & - & 0,64 & 0,67 & 0,71 & 0,84 \\
6. Motivación intrínseca & 3,25 & 1,24 & 0,69 & 0,51 & 0,68 & 0,49 & 0,70 & - & 0,61 & 0,73 & 0,85 \\
7. Satisfacción laboral & 3,26 & 1,28 & 0,63 & 0,29 & 0,57 & 0,38 & 0,72 & 0,65 & - & 0,82 & 0,90 \\
\hline
\end{tabular}

Nota: Los valores bajo la diagonal corresponden a la proporción heterorasgo-monorasgo entre factores. Negrita: $\mathrm{p}<0,01 ;$ cursiva: $p<0,05$. 
ostrar si la pasión por el trabajo constituye un constructo distinguible del flow y de la implicación en el trabajo. Por otro lado, determinar su utilidad pragmática y estadística en la explicación de la satisfacción laboral.

En lo que hace al primer objetivo, la exploración preliminar mediante el análisis ESEM indicó que un modelo de medida integrado por siete factores latentes correlacionados, con sus respectivos ítems como variables observables y los correspondientes errores de medición, no difería significativamente de la matriz de covarianza de los datos. Todos los ítems pesaron notablemente más en los factores latentes esperados. Aun cuando las saturaciones cruzadas no fueron nulas, en ningún caso superaron el límite esta- blecido por la literatura especializada (Lloret et al., 2017; Marsch et al., 2014). Sumado a esto, los valores obtenidos en los índices AVE, su raíz cuadrada y la proporción HTMT mostraron que más del $50 \%$ de sus varianzas son atribuibles a sus respectivos indicadores, y que cada constructo comparte más varianza con sus propios indicadores que con los demás (Bagozzi, \& Yi, 2012; Henseler et al., 2015). El análisis de correlaciones efectuado mostró, a su vez, vinculaciones significativas entre todas las variables estudiadas; las que en ningún caso fueron próximas a 1 . En conjunto, estos resultados demuestran fehacientemente la validez discriminante de la pasión por el trabajo, proporcionando apoyo total a la primera hipótesis.

\section{Tabla 3}

Estimaciones del ajuste y coeficientes de regresión de los modelos contrastados.

\begin{tabular}{|c|c|c|c|c|c|c|c|c|}
\hline Predictores & $\beta$ & $t$ & $p$ & $r_{\mathrm{sp}}$ & $R^{2}$ & $\Delta R^{2}$ & AIC & $f^{2}$ \\
\hline \multicolumn{9}{|l|}{ Modelo 1} \\
\hline Disfrute & 0,55 & 12,95 & 0,000 & 0,31 & & & & \\
\hline Motivación intrínseca & 0,47 & 10,02 & 0,000 & 0,25 & & & & \\
\hline Absorción & 0,27 & 5,62 & 0,000 & 0,18 & & & & \\
\hline Implicación en el trabajo & 0,38 & 9,93 & 0,000 & 0,24 & 0,531 & $0,531^{* * *}$ & 85,18 & 1,13 \\
\hline \multicolumn{9}{|l|}{ Modelo 2} \\
\hline Disfrute & 0,51 & 12,28 & 0,000 & 0,29 & & & & \\
\hline Motivación intrínseca & 0,42 & 9,65 & 0,000 & 0,23 & & & & \\
\hline Absorción & 0,20 & 5,13 & 0,000 & 0,14 & & & & \\
\hline Implicación laboral & 0,33 & 9,32 & 0,000 & 0,20 & & & & \\
\hline Pasión armoniosa & 0,49 & 10,96 & 0,000 & 0,27 & & & & \\
\hline Pasión obsesiva & 0,15 & 3,82 & 0,000 & 0,18 & 0,579 & $0,048^{* * *}$ & 79,26 & 1,37 \\
\hline
\end{tabular}

Nota: $\beta=$ Coeficiente de regresión estandarizado; $r_{\mathrm{sp}}=$ Correlación semiparcial; $R^{2}=$ Correlación múltiple al cuadrado; $\Delta R^{2}=$ Incremento del cambio del coeficiente de correlación múltiple al cuadrado; ${ }^{* * *}=p<0,001$; AIC = Criterio de Información de Akaike; $f_{=}$Coeficiente $\mathrm{f}^{2}$ de Cohen para el tamaño de efecto.

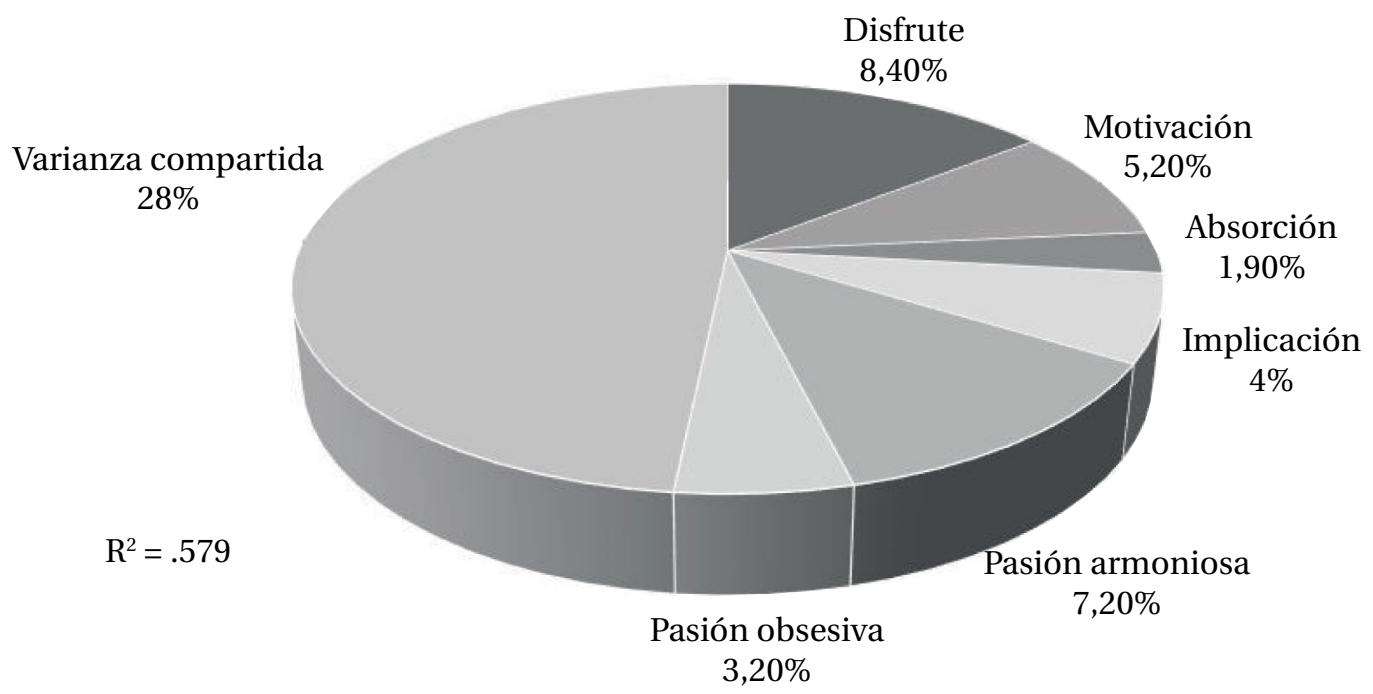

Figura

Porcentajes de varianza explicada de satisfacción laboral específica y compartida. 
Un análisis pormenorizado de la dirección de las correlaciones entre los constructos muestra asociaciones positivas entre la pasión y la implicación laboral. No obstante, cabe destacar que en el presente estudio tales vinculaciones resultaron superiores para la pasión obsesiva. Este hallazgo demuestra la relevancia que el trabajo tiene para la autoestima y la identidad de estos empleados, sintonizando así con las conclusiones de recientes comunicaciones científicas (Birkeland, \& Buch, 2015; Lalande et al., 2017; Loriol, 2017). Paralelamente, las relaciones positivas encontradas entre flow y pasión sintoniza con las conclusiones de investigaciones previas (Carpentier, Mageau, \& Vallerand, 2012; Curran, et al., 2015), sugiriendo que los individuos apasionados se encuentran completamente abocados a su trabajo. Los altos montos de esfuerzo y dedicación invertidos en la ejecución de sus tareas explicarían la mayor absorción y motivación intrínseca, así como la posibilidad de disfrute y satisfacción mientras estén involucrados en su trabajo; aún, entre los obsesivamente apasionados. Finalmente, del presente estudio se desprende que la pasión por el trabajo en sus dos variantes se asocia significativamente a la satisfacción. No obstante, tales vinculaciones son notablemente superiores para la pasión armoniosa. Dicho hallazgo resulta congruente con las conclusiones de recientes publicaciones (Curran et al., 2015; Orgambídez-Ramosa et al., 2014Salesi, \& Omar, en prensa ${ }^{3}$; Thorgren, Wincent, \& Sirén, 2013; Zito, \& Colombo, 2017), que subrayan que las personas armoniosamente apasionadas son más proclives que sus contrapartes, los obsesivos, a desarrollar estados y actitudes positivas.

En cuanto al segundo objetivo perseguido por esta investigación, cabe señalar que el análisis de regresión jerárquica realizado confirmó la validez predicativa e incremental de la pasión. En este sentido, luego de controlar los efectos del disfrute, la motivación intrínseca, la absorción y la implicación, la pasión por el trabajo armoniosa y obsesiva realizan un incremento significativo a la varianza explicada de la satisfacción laboral. Tales hallazgos proporcionan apoyo empírico tanto a la hipótesis 2, que adelantaba el rol predictor del flow y de la implicación; como a la hipótesis 3 , que anticipaba la validez predictiva-incremental de la pasión por el trabajo.

En sintonía con los hallazgos aquí obtenidos, recientes comunicaciones científicas (Akhtar, Nadir,
\& Nadir, 2016; Bayraktar et al., 2017; Konradt, \& Garbers, 2016) destacan que la implicación y el flow mantienen un nexo inexorable con la satisfacción laboral, explicando porciones significativas de su variabilidad. Asimismo, distintos estudios comprueban la validez predictiva e incremental de la pasión en la explicación de diversas actitudes y comportamientos. En este sentido, por ejemplo, Birkeland y Buch (2015) encontraron que, luego de controlar los efectos de la adicción al trabajo y del engagement, la pasión por el trabajo en sus dos vertientes predice significativamente el burnout y la satisfacción con la vida. Del mismo modo, Houlfort et al. (2014) halló que la pasión armónica y obsesiva explican las intenciones de renunciar y la satisfacción de los empleados más allá de sus niveles de motivación en el trabajo. En la misma línea, Thorgren et al. (2013) señalaron que, más allá de los pensamientos sobre la tarea fuera y dentro de la jornada laboral, la pasión por el trabajo predice significativamente la satisfacción.

\section{Limitaciones, fortalezas e implicancias prácticas del estudio}

Entre las principales limitaciones de la investigación realizada hay que citar la representatividad de la muestra; la que, al haber sido seleccionada en forma no probabilística, impide generalizar los resultados al conjunto de la población. Sin embargo, vale destacar que con el propósito de minimizar este inconveniente se procuró que la muestra en estudio incluyera empresas e instituciones de los más diferentes rubros y niveles de complejidad organizacional. Del mismo modo, el diseño transversal utilizado impide cualquier inferencia de causalidad estricta entre las variables estudiadas. Por lo que sería valioso que futuras investigaciones emplearan diseños longitudinales con miras a establecer el orden causal de las relaciones aquí encontradas. Por último, dado que en el presente estudio se han utilizado instrumentos de autoinforme para evaluar las variables bajo análisis, las respuestas podrían encontrarse contaminadas tanto por la tendencia a aparecer mejor como por otros componentes subjetivos. Para superar esta limitación sería recomendable que futuros estudios incluyeran una medida de deseabilidad social como recurso de validez interna.

Más allá de estas limitaciones, el trabajo realizado contribuye a la literatura existente en dos sen-

${ }^{3}$ Salessi, S. \& Omar, A. (en prensaa). Validación Escala Pasión por el Trabajo: Un estudio instrumental con trabajadores argentinos. Psykhé. 
tidos. Por un lado, esta investigación constituye la primera tentativa de analizar la validez discriminante, predictiva e incremental del constructo pasión por el trabajo con relación al flow y a la implicación laboral, por lo que representa una contribución genuina a la literatura organizacional. Por otro lado, demuestra que la pasión se erige como variable clave en la predicción de actitudes laborales trascendentes; desde el momento que realiza una contribución sustancial a la explicación de la satisfacción laboral.

Del estudio realizado se desprenden algunas implicancias prácticas, tanto para los estudiosos del comportamiento organizacional, como para gerentes y directivos de recursos humanos. Para los primeros, los hallazgos confirman que la inclusión de la pasión por el trabajo en un modelo que pretenda explicar resultados organizacionalmente relevantes puede mejorar significativamente la predicción, logrando así mayores niveles de precisión y reducción del error en las estimaciones. Para los segundos, los resultados iluminan el impacto favorable de la pasión en la satisfacción de los empleados. Por ende, las organizaciones preocupadas por garantizar el bienestar de sus miembros deberían concentrarse en la creación de un entorno capaz de contribuir al florecimiento del flow, de la implicación y, especialmente, de la pasión por el trabajo. En este sentido, se ha demostrado (Perrewé et al., 2014; Vallerand, 2015) que, por ejemplo, promover una cultura que afiance los lazos interpersonales y fomente un sentido de pertenencia, formar a quienes ocupen posiciones clave como líderes transformacionales y, diseñar puestos que brinden mayor autonomía en la planificación del trabajo son intervenciones promotoras de actitudes y experiencias positivas Tales acciones podrían sentar las bases de una organización saludable y positiva, favoreciendo mayores niveles de satisfacción y compromiso entre sus miembros (Warren et al., 2017).

\section{Referencias}

Akhtar, Z., Nadir, P., \& Nadir, H. (2016). Job satisfaction and job involvement among private and government bank employees. Indian Journal of Health and Wellbeing, 7(2), 236-239.

Astakhova, M., \& Porter, G. (2015). Understanding the work passion-performance relationship: The mediating role of organizational identification and moderating role of fit at work. Human Relations, 68, 1315-1346. https://doi.org/10.1177/0018726714555204.

Ato, M., López, J. J., \& Benavente, A. (2013). Un sistema de clasificación de los diseños de investigación en psicología. Anales de Psicología, 29(3), 1038-1059. https://doi.org/10.6018/analesps.29.3.178511

Bagozzi, R. \& Yi, Y. (2012). Specification, evaluation, and interpretation of structural equation models. Journal of the Academy of Marketing Science, 40, 8-34. https://doi.org/10.1007/s11747-011-0278-x

Bakker, A. (2008). The work-related flow inventory: Construction and initial validation of the WOLF. Journal of Vocational Behaviour 72, 400-414. https://doi.org/10.1016/j.jvb.2007.11.007

Bayraktar, C., Araci, O., Karacay, G. \& Calisir, F. (2017). The mediating effect of rewarding on the relationship between employee involvement and job satisfaction. Human Factors \& Ergonomics in Manufacturing \& Service Industries, 27(1), 45-52. https://doi.org/10.1002/hfm.20683

Birkeland, I. \& Buch, R. (2015). The dualistic model of passion for work: Discriminate and predictive validity with work engagement and workaholism. Motivation \& Emotion. 39, 392-408. https://doi.org/10.1007/s11031-014-9462-x

Carpentier,J., Mageau. G., \&Vallerand, R. (2012). Ruminations and flow: Why do people with a more harmonious passion experience higher well-being? Journal of Happiness Studies, 13, 501-518. https://doi.org/10.1007/s10902-011-9276-4.

Cheng, Z. (2014). The effects of employee involvement and participation on subjective wellbeing: Evidence from urban China. Social Indicators Research, 118(2), 457-483. https://doi.org/10.1007/s11205-013-0430-8

Curran, T., Hill, A., Appleton, P., Vallerand, R., \& Standage, M. (2015). The psychology of passion: A meta-analytical review of a decade of research on intrapersonal outcomes. Motivation \& Emotion, 39(5), 631-655. https:// doi.org/10.1007/s11031-015-9503-0

Engeser, S. \& Baumann, N. (2016). Fluctuation of flow and affect in everyday life: A second look at the paradox of work. Journal of Happiness Studies, 17, 105-124. https://doi.org/10.1007/s10902-014-9586-4 
Farrell, A. (2010). Insufficient discriminant validity: A comment on Bove, Pervan, Beatty and Shiu (2009). Journal of Business Research, 63(3), 324-327. https:// doi.org/10.1016/j.jbusres.2009.05.003

Gorgievski, M. \& Bakker, A. (2010). Passion for work: Work engagement versus workaholism. In A. Simon (Ed), Handbook of employee engagement: Perspectives, issues, research and practice (pp. 264-271). New York, NY: Edward Elgar.

Hair, J. E., Black, W. C., Babin, B. J., Anderson, R. E., \& Tatham, R. L. (2010). Multivariate data analysis (6th ed.). Upper Saddle River, NJ: Pearson-Prentice Hall.

Harter, J. K., \& Schmidt, F. L. (2008). Conceptual versus empirical distinctions among constructs: Implications for discriminant validity. Industrial and Organizational Psychology: Perspectives on Science and Practice, 1, 36-39. https://doi.org/10.1111/j.1754-9434200700004.x

Henseler, J., Ringle, C. M., \& Sarstedt, M. (2015). A new criterion for assessing discriminant validity in variance-based structural equation modelling. Journal of the Academy of Marketing Science, 43(1), 115-135. https://doi.org/10.1007/s11747-014-0403-8

Houlfort, N. Philippe, F, Vallerand, R., \& Ménard, J. (2014). On passion and heavy work investment: Personal and organizational outcomes. Journal of Managerial Psychology, 29, 25-45. https://doi.org/10.1108/JMP-06-2013-0155

Hunsley,J.\&Meyer,G.(2003).Theincremental validityof psychologicaltestingand assessment:Conceptual, methodological and statistical issues. Psychologica Assessment, 15(4), 446-455. https:// doi.org/10.1037/1040-3590.15.4.446

Ilies, R., Wagner, D., Wilson, K., Ceja, L., Johnson, M., DeRue, S., \& Ilgen, D. (2017). Flow at work and basic psychological needs: Effects on well-being. Applied Psychology: An International Review, 66(1), 3-24. https://doi.org/10.1111/apps.12075

Johari, J. \& Yahya, K. (2016). Job characteristics, work involvement and job performance of public servants. European Journal of Training \& Development, 40(7), 554-575. https://doi.org/10.1108/EJTD-07-2015-0051

Kanungo, R. N. (1982). Measurement of job and work involvement. Journal of Applied Psychology, 67, 341-349. https://doi.org/10.1037/0021-9010.67.3.341

Konradt, U. \& Garbers, Y. (2016). The role of job and family involvement for satisfaction in job and family: A longitudinal study. Zeitschrift für Psychologie, 224(1), 15-24. https://doi.org/10.1027/2151-2604/a000234

Lalande, D., Vallerand, R., Lafrenière, M., Verner-Filion, J., Laurent, F., Forest, J., \& Paquet, Y. (2017). Obsessive passion: A compensatory response to unsatisfied needs. Journal of Personality, 85,119-280. https://doi.org/10.1111/jopy.12229

Le, H., Schmidt, F., Harter, J., \& Lauver, K. (2010). The problem of empirical redundancy of constructs in organizational research: An empirical investigation. Organizational Behavior and Human Decision Processes 112, 112125. https://doi.org/10.1016/j.obhdp.2010.02.003

Loriol, M. (2017). The collective regulation of Smac workers' passion and involvement. Sociologia del Lavoro, 145, 168-183. https://doi.org/10.3280/SL2017-1450010

Lloret, S., Ferreres, A., Hernández, A., \& Tomás, I. (2017). The exploratory factor analysis of items: Guided analysis based on empirical data and software. Anales de Psicología, 33(2), 417-432. https://doi.org/10.6018/analesps.33.2.270211

Maeran, R. \& Cangiano, F. (2013). Flow experience and job characteristics: Analyzing the role of flow in job satisfaction. TPM-Testing, Psychometrics, Methodology in Applied Psychology, 20(1), 13-26. https:// doi.org/10.4473/TPM20.1.2

Marsh, H., Morin, A., Parker, P., \& Kaur, G. (2014). Exploratory structural equation modeling: An integration of the best features of exploratory and confirmatory factor analysis. Annual Review of Clinical Psychology, 10, 85-110. https://doi.org/10.1146/annurev-clinpsy-032813-153700

Marsh, H., Vallerand, R., Lafreniere, M., Parker, P., Morin, A., Carbonneau, N. et al. (2013). Passion: Does one scale fit all? Construct validity of two-factor Passion Scale and psychometric invariance over different activities and languages. Psychological Assessment, 25, 796-809. https://doi.org/10.1037/a0032573

Merino, S. C. A. (2014). Validez incremental del Test Gestáltico de Bender Modificado en niños iniciando que inician el primer grado. Avances en Psicología Latinoamericana, 32(2), 275-286. https://doi.org/10.12804/apl32.2.2014.07

Morrow, P. C. (1983). Concept redundancy in organizational research: The case of work commitment. Academy of Management Review, 8, 486-500. https://doi.org/10.5465/amr.1983.4284606

Muthén, L.K., \& Muthén, B.O. (2014). Mplus user’s guide (7th ed.). Los Angeles, CA: Muthén \& Muthén. 
Orgambídez-Ramos, A., Borrego-Alés, Y., \& Gonçalves, G. (2014). Passionate workers: A Spanish adaptation of the Passion Scale. Revista de Psicología del Trabajo y de las Organizaciones, 30(2), 43-48. https://doi.org/10.1016/j.rpto.2014.06.005.

Perrewé, P., Hochwarter, W., Ferris, G., Mcallister, C., \& Harris, J. (2014). Developing a passion for work passion: Future directions on an emerging construct. Journal of Organizational Behaviour, 35, 145-150. https://doi.org/10.1002/job.1902

Reichwein-Zientek, L., \& Thompson, B. (2006). Commonality analysis: Partitioning variance to facilitate better understanding of data. Journal of Early Intervention, 28(4), 299-307.https:// doi.org/10.1177/ 105381510602800405

Rousseau, D. M. (2007). A sticky, leveraging, and scalable strategy for high-quality connections between organizational practice and science. Academy of Management Journal, 50, 1037-1042. https:// doi.org/1010.5465/amj.2007.27155539

Ryan, R., \& Deci, E. (2016). Self-determination theory basic psychological needs in motivation, development, and wellness. New York, NY: Guilford.

Sackett P., Dahlke J., Shewach O., \& Kuncel N. (2017). Effects of predictor weighting methods on incremental validity. The Journal of Applied Psychology, 102(10), 1421-1434. https:// doi.org/10.1037/apl0000235

Salessi, S. \& Omar, A. (2016). Satisfacción laboral genérica: Propiedades psicométricas de una escala para medirla. Revista Alternativas en Psicología, 34, 93-108

Schwab, D. E. (1980). Construct validity in organizational behavior. In B. M. Staw \& L. L. Cummings (Eds.). Research in organizational behavior (Vol. 2, pp. 3-43). Greenwich, CT: JAI.

Tabachnick, B. G., \& Fidell, L. S (2013). Using Multivariate Statistics (6th ed.). Boston, MA: Pearson.

Taris, T., Van Beek, I., \& Schaufeli, W.B. (2014). The beauty versus the beast: On the motives of engaged and workaholic employees. In I. Harpaz, \& R. Snir (Eds), Heavy work investment: Its nature, sources, outcomes, and future directions (pp. 121-138.). New York, NY: Taylor \& Francis/Routledge.

Thorgren, S., Wincent, J., \& Sirén, C. (2013). The influence of passion and work-life thoughts on work satisfaction. Human Resource Development Quarterly, 24, 469-492. 24. https://doi.org/10.1002/hrdq.21172.

Vallerand, R. (2015). The psychology of passion: A dualistic model. Oxford, UK: Oxford University Press.

Vallerand, R., Blanchard, C., Mageau, G., Koestner, R., Ratelle, C., Le'onard, M. et al. (2003). Les passions de l'a^me: On obsessive and harmonious passion. Journal of Personality and Social Psychology, 85, 756-767. https://doi.org/10.1037/0022-3514.85.4.756

Vallerand, R. J., \& Houlfort, N. (2003). Passion at work: Toward a new conceptualization. In S. W. Gilliland, D. Steiner, \& D. Skarlicki (Eds.), Emerging perspectives on values in organizations (pp. 175-204). Greenwich, CT: Information Age.

Vallerand, R., \& Verner-Filion, J., (2013). Making peoples' life most worth living: On the importance of passion for positive psychology. Terapia Psicológica, 31(1), 35-48.

Warren, M., Donaldson, S., \& Luthans, F. (2017). Taking positive psychology to the workplace: Positive organizational psychology, positive organizational behavior, and positive organizational scholarship. In M. Warren, \& S. Donaldson (Eds.), Scientific advances in positive psychology (pp. 195-227). Santa Barbara, CA: Praeger/ABC-CLIO.

Zigarmi, D., Nimon, K., Houson, D., Witt, D., \& Diehl, J. (2009). Beyond engagement: Toward a framework and operational definition for employee work passion. Human Resource Development Review, 8, 300-326. https:// doi.org/10.1177/1534484309338171

Zito, M. \& Colombo, L. (2017). The Italian version of the Passion for Work Scale: First psychometric evaluations. Journal of Work and Organizational Psychology 33, 47-53. https://doi.org/10.1016/j.rpto.2017.01.003

\section{Solana Salessi}

Pontificia Universidad Católica Argentina, Facultad de Derecho y Ciencias Sociales del Rosario Universidad Nacional de Rafaela, Dpto de Educación, Cultura y Conocimiento. Consejo Nacional de Investigaciones Científicas y Técnicas.

E-mail: solanasalessi@gmail.com 


\section{Alicia Omar}

Integrante do Consejo Nacional de Investigaciones Científicas y Técnicas (Conicet).

E-mail:aomar@yahoo.com

Endereço para envio de correspondência:

Argentina, Av. Pellegrini 3314 (2000) Rosario - Santa Fe - Argentina.

E-mail: solanasalessi@uca.edu.ar

Recebido 16/11/2017

Aprovado 06/06/2018

Received 11/16/2017

Approved 06/06/2018

Recibido 16/11/2017

Aceptado 06/06/2018

Cómo citar: Salessi, S., \& Omar, A. (2018). Pasión por el trabajo: Evidencias de validez discriminante, predictiva e incremental. Psicologia: Ciência e Profissão, 38(3), 522-536. https://doi.org/10.1590/1982-37030004752017

How to cite: Salessi, S., \& Omar, A. (2018). Work passion: Evidence of discriminant, predictive and incremental validity. Psicologia: Ciência e Profissão, 38(3), 522-536. https:// doi.org/10.1590/1982-37030004752017

Como citar: Salessi, S., \& Omar, A. (2018). Paixão pelo trabalho: Evidências de validade discriminante, predictiva e incremental. Psicologia: Ciência e Profissão, 38(3), 522-536. https://doi.org/10.1590/1982-37030004752017 